\title{
Glycinergic transmission: physiological, developmental and pathological implications
}

\author{
Robert J. Harvey ${ }^{*}$ and Jean-Michel Rigo ${ }^{2}$ \\ Department of Pharmacology, The School of Pharmacy, London, UK \\ 2 Institute of Biomedical Research, Hasselt University and transnationale Universiteit Limburg, Diepenbeek, Belgium \\ ${ }^{*}$ Correspondence: robert.harvey@pharmacy.ac.uk
}

The last few years have seen remarkable developments in our understanding of the physiology, pharmacology and genetics of inhibitory glycinergic synapses. In part, this has been due to the development of new resources such as specific antisera recognizing glycine receptor (GlyR) and transporter (GlyT) subtypes, but also the characterization of new mouse, zebrafish and bovine genetic models of glycinergic dysfunction. What is also evident is the high quality and impact of the research conducted in this field. This is reflected in the reviews and research articles in this Special Issue entitled "Glycinergic transmission: physiological, developmental and pathological implications".

The study of inhibitory synaptic transmission has a long and illustrious history, as documented by Callister and Graham (2010). Key in vivo experiments on spinal glycinergic synapses conducted in the 1950s and 1960s helped to define key concepts in chemical neurotransmission and the distinct pharmacological and electrophysiological properties of what we now know to be inhibitory GlyRs containing the $\alpha 1$ and $\beta$ subunits. This major adult GlyR isoform predominates in the spinal cord and brainstem (Baer et al., 2009) and has a major role the control of spinal motor reflex circuits. Defects in the corresponding genes, GLRA1 and GLRB, result in an inherited motor disorder in humans known as hyperekplexia, characterized by neonatal hypertonia and an exaggerated startle reflex. Modern genetics techniques (Davies et al., 2010) have revealed that hyperekplexia is best thought of as a synaptopathy, since mutations in SLC6A5 - encoding the presynaptic glycine transporter GlyT2 - can also cause startle disease. Other GlyR subtypes, such as those containing the $\alpha 2, \alpha 3$ and $\alpha 4$ subunits, may play more diverse biological roles in retinal circuitry (Wässle et al., 2009) and central inflammatory pain sensitization (Harvey et al., 2009). GlyR $\alpha 2$ and $\alpha 3$ subunit transcripts are also unusual in that they undergo both alternative splicing and cytidine to uracil RNA editing (C to U), resulting in a proline to leucine substitution $(\mathrm{P} 185 \mathrm{~L}$ in $\alpha 3, \mathrm{P} 192 \mathrm{~L}$ in $\alpha 2$ ) that confers high agonist sensitivity and pharmacology to "edited" GlyRs (Legendre et al., 2009). GlyR transcript editing may promote the generation of sustained chloride conductances associated with tonic inhibition and is modulated by brain lesions, suggesting a possible involvement with pathogenic processes. These "orphan" GlyR subtypes may also have key roles in peripheral tissues, since GlyRs have been located on sperm and neutrophils. However, in renal, liver and endothelial cells, where glycine protects from cell death, caution should be applied in attributing these functions to classical GlyRs and GlyTs (Van den Eynden et al., 2009). Certainly, not all cell types that express GlyR subunit mRNAs or polypeptides exhibit GlyR-mediated membrane conductance changes. It is also noteworthy that NMDA receptors composed of the NR1 and NR3 subunits lack glutamate-binding sites and can be activated by glycine alone. It is therefore imperative to understand the synaptic location and pharmacology of this "excitatory" GlyR (Madry et al., 2010).

So what does the future hold for the study of glycinergic transmission? Certainly, GlyRs have a far richer pharmacology than has been appreciated until now. The advent of high throughput screening techniques using anion-sensitive EYFP has enabled automated electrophysiology approaches to be applied in the search for new GlyR-active compounds and subtype-specific modulators (Gilbert et al., 2009). In addition, further study of spontaneous or knockout models of GlyR and GlyT dysfunction has the potential to reveal new roles for these synaptic proteins. In particular, the biological roles of the GlyR $\alpha 2$ and $\alpha 4$ subtypes still remain enigmatic. The embryonic/neonatal GlyR $\alpha 2$ subtype has previously been linked to roles in synaptogenesis, cell fate/ paracrine transmitter release in the developing cortex/spinal cord and retinal photoreceptor development. It was therefore somewhat surprising that Glra2 knockout mice did not show a clear behavioral phenotype. This is most likely due to the "rewiring" of neuronal circuits during development allowing compensatory mechanisms to mask certain phenotypes. For example, the loss of GlyR $\alpha 3$ in a knockout model results in both presynaptic and postsynaptic compensation in the spinal cord. Lamina II synapses that typically express both $\alpha 3 \beta$ GlyRs show an elevated glycine release probability, with no changes in quantal content onto $\alpha 1 \beta$ GlyRs, which continue to mediate synaptic transmission. Phenotypes revealed to date in Glra3 knockout mice have exclusively been linked to G-protein coupled receptor pathways influencing PKA-mediated phosphorylation of GlyR $\alpha 3$. In fact, these were only evident because $\alpha 1 \beta$ GlyRs are not modulated by PKA phosphorylation. Whilst new knock-in models expressing dominant-negative mutations might overcome this issue, other model organisms will undoubtedly play an important role. For example, zebrafish have a full complement of GlyR and GlyT genes and are amenable to developmental and genetic analysis using $N$-ethyl- $N$-nitrosourea (ENU) mutagenesis, gene-traps and rapid targeted gene "knockdown" using antisense morpholinos (Ganser and Dallman 2009; Chalphin and Saha 2010; Hirata et al., 2010). Curiously, the gene encoding GlyR $\alpha 4$ is thought to be a pseudogene in humans due to a stop codon in GLRA4 exon 9, causing a protein truncation between membrane-spanning domains M3 and M4. However, this finding may need revisiting in the light of recent resequencing studies that highlight that certain genes on the $\mathrm{X}$ chromosome are intact in some individuals but contain non-sense or frameshift changes in other apparently normal 
control subjects. It would therefore seem that some genes that are apparently inert in some humans may be active in others. It is also certain that additional defects involving glycinergic transmission remain to be identified. Not all cases of hyperekplexia can be explained by mutations in the genes encoding the adult GlyR $\alpha 1 \beta$ isoform or GlyT2, implying that researchers are either missing mutations in important gene regulatory elements, or in other genes involved in the formation/function of glycinergic synapses (Davies et al., 2010). In addition, several hyperekplexialike syndromes in animals remain unresolved, such as inherited myoclonus in Peruvian Paso horses and familial reflex myoclonus

\section{REFERENCES}

Baer, K., Waldvogel, H. J., Faull, R. L., and Rees, M. I. (2009). Localisation of glycine receptors in the human forebrain, brainstem, and cervical spinal cord: an immunohistochemical review. Front. Mol. Neurosci. 2:25. doi:10.3389/ neuro.02.025.2009.

Callister, R. J., and Graham, B. A. (2010). Early history of glycine receptor biology in mammalian spinal cord circuits. Front. Mol. Neurosci. 3:13. doi:10.3389/fnmol.2010.00013.

Chalphin, A. V., and Saha, M. S. (2010). The specification of glycinergic neurons and the role of glycinergic transmission in development. Front. Mol. Neurosci. 3:11. doi:10.3389/ fnmol.2010.00011.

Davies, J. S., Chung, S., Thomas, R. H., Robinson,A.,Hammond,C.L., Mullins, J. G., Carta, E., Pearce, B. R., Harvey, K., Harvey, R. J., and Rees, M. I. (2010). The glycinergic system in human startle disease: a genetic screening approach. Front. Mol. Neurosci. 3:8. doi:10.3389/fnmol.2010.00008.

Dumoulin, A., Triller, A., and Kneussel, M. (2010). Cellular transport and membrane dynamics of the glycine receptor. Front. Mol. Neurosci. 2:28. doi:10.3389/neuro.02.028.2009.

Ganser, L. R., and Dallman, J. E. (2009). Glycinergic synapse development, plasticity, and homeostasis in zebrafish. Front. Mol. Neurosci. 2:30. doi:10.3389/neuro.02.030.2009.

Gilbert, D. F., Islam, R., Lynagh, T., Lynch, J. W., and Webb, T. I. (2009). High throughput techniques for discovering new glycine receptor modulators and their binding sites. Front. Mol. Neurosci. 2:17. doi:10.3389/ neuro.02.017.2009.

Harvey, V. L., Caley, A., Müller, U. C., Harvey, R. J., and Dickenson, A. H. (2009). A selective role for $\alpha 3$ subunit glycine receptors in inflammatory pain. Front. Mol. Neurosci. 2:14. doi:10.3389/neuro.02.014.2009.

in labrador retrievers. Lastly, although we know much about the cellular transport and membrane dynamics of GlyRs (Dumoulin et al., 2010) - mediated in part by the multifunctional protein gephyrin -our knowledge concerning proteins associated with GlyRs and GlyTs is still painfully thin. The development of reliable antibodies that function in immunoprecipitation and the application of modern proteomics techniques to the study of glycinergic synapses is therefore a priority for the future.

We thank all contributors for their interesting and informative articles and the reviewers for their constructive and thoughtful suggestions.

Hirata, H., Carta, E., Yamanaka, I., Harvey, R. J., and Kuwada, J. Y. (2010). Defective glycinergic synaptic transmission in zebrafish motility mutants. Front.Mol.Neurosci. 2:26. doi:10.3389/ neuro.02.026.2009.

Legendre, P., Förstera, B., Jüttner, R., and Meier, J. C. (2009). Glycine receptors caught between genome and proteome - functional implications of RNA editing and splicing. Front. Mol. Neurosci. 2:23. doi:10.3389/ neuro.02.023.2009.

Madry, C., Betz, H., Geiger, J., and Laube, B. (2010). Potentiation of glycine-gated NR1/NR3A NMDA receptors relieves $\mathrm{Ca} 2+$-dependent outward rectification. Front. Mol. Neurosci. 3:6. doi:10.3389/ fnmol.2010.00006.

Van den Eynden, J.,Saheb Ali, S., Horwood, N., Carmans, S., Brône, B., Hellings, N., Steels, P., Harvey, R. J., and Rigo, J. (2009). Glycine and glycine receptor signalling in non-neuronal cells.
Front. Mol. Neurosci. 2:9. doi:10.3389/ neuro.02.009.2009.

Wässle, H., Heinze, L., Ivanova, E., Majumdar, S., Weiss, J., Harvey, R. J., and Haverkamp, S. (2009). Glycinergic transmission in the mammalian retina. Front. Mol. Neurosci. 2:6. doi:10.3389/ neuro.02.006.2009.

Received : 03 August 2010; accepted: 03 August 2010; published online: 19 August 2010

Citation: Harvey RJ and Rigo J-M (2010). Glycinergic transmission: physiological, developmental and pathological implications. Front. Mol. Neurosci. 3:115. doi:10.3389/fnmol.2010.00115

Copyright $\odot 2010$ Harvey and Rigo. This is an open-access article subject to an exclusive license agreement between the authors and the Frontiers Research Foundation, which permits unrestricted use, distribution, and reproduction in any medium, provided the original authors and source are credited. 